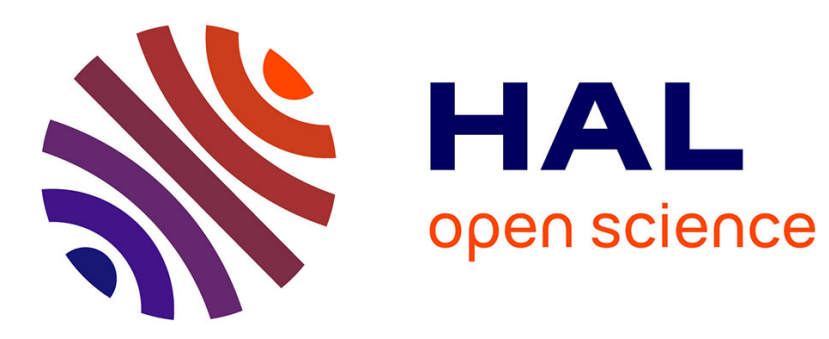

\title{
Responsive Self-assemblies based on Fatty acids
}

Anne-Laure Fameau, Audrey Arnould, Arnaud Saint-Jalmes

\section{To cite this version:}

Anne-Laure Fameau, Audrey Arnould, Arnaud Saint-Jalmes. Responsive Self-assemblies based on Fatty acids. Current Opinion in Colloid \& Interface Science, 2014, 19 (5), pp.471-479. 10.1016/j.cocis.2014.08.005 . hal-01062933

\section{HAL Id: hal-01062933 https://hal.science/hal-01062933}

Submitted on 8 Oct 2014

HAL is a multi-disciplinary open access archive for the deposit and dissemination of scientific research documents, whether they are published or not. The documents may come from teaching and research institutions in France or abroad, or from public or private research centers.
L'archive ouverte pluridisciplinaire HAL, est destinée au dépôt et à la diffusion de documents scientifiques de niveau recherche, publiés ou non, émanant des établissements d'enseignement et de recherche français ou étrangers, des laboratoires publics ou privés. 


\section{Responsive Self-assemblies based on Fatty acids}

\section{Anne-Laure Fameau ${ }^{1 *}$, Audrey Arnould ${ }^{1}$ and Arnaud Saint-Jalmes ${ }^{2}$}

${ }^{1}$ UR1268 Biopolymères Interactions Assemblages Institut National de la Recherche Agronomique, rue de la Géraudière, F-44316 Nantes cedex 3, France.

${ }^{2}$ Institut de Physique de Rennes, UMR CNRS 6251 -Université Rennes 1, Rennes, France.

- corresponding author : Anne-Laure Fameau

- tel: +33240675083

- email: anne-laure.fameau@nantes.inra.fr

\section{Graphical Abstract}

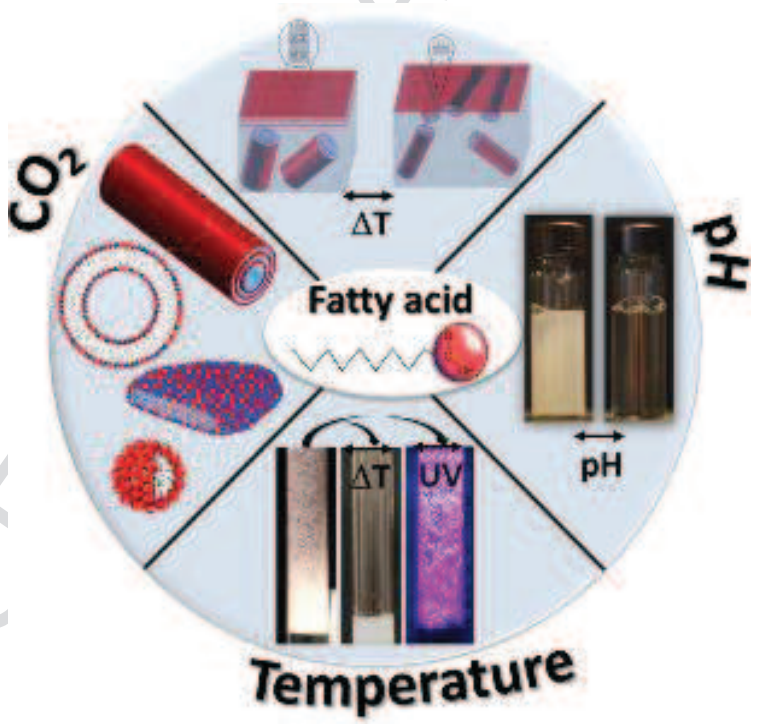

Keywords: Fatty acid, self-assembly, responsive surfactant, foam, interface, rheology

\section{Highlights:}

- Review of fatty acid as responsive surfactant

- Correlation between molecular state and self-assemblies structural transitions triggered by $\mathrm{pH}, \mathrm{CO}_{2}$ and temperature

- Link between self-assemblies based on fatty acids and corresponding macroscopic properties under stimuli

- Chain melting process as prerequisite to obtain thermoresponsive interfaces and foams 


\begin{abstract}
Fatty acids are anionic surfactants under their deprotonated forms. They are surfactants with both biodegrability and low toxicity. Fatty acid molecules can self-assemble under various shapes in aqueous solution. These self-assembled structures can respond to stimuli such as $\mathrm{pH}, \mathrm{CO}_{2}$ and temperature due to changes occurring at the molecular level. These specificities make them surfactants of special interest to tune the properties at the macroscopic scale. The aim of this article is to review the recent advances in the creation and in the understanding of responsive self-assemblies obtained from fatty acid molecules in aqueous solution. The links between the microscopic, mesoscopic and macroscopic scales are described. The alkyl chain melting phenomenon triggered by temperature at the molecular level leading to thermoresponsive interfaces and foams at the macroscopic scale is highlighted.
\end{abstract}

\title{
1. Introduction
}

Soft materials which respond to stimuli are on the leading edge of materials research and have recently been a subject of growing interest to many scientists [1]. The macroscopic responsiveness relies on the ability to react at microscopic or mesoscopic scales. Stimuliresponsive surfactants that can change their structure in response to a trigger such as $\mathrm{pH}$, temperature, light or magnetic field have attracted great attention due to their versatile applications in various fields (e.g.pharmaceutical, biomedical, nanotechnology, etc.) [2]. A change in the molecular structure of the surfactant activated by stimuli can affect the selfassembled structure in water and the interfacial activity, which can in turn tune the properties at the macroscopic scale such as viscosity, emulsion and foam stability [2].

In the current context of sustainable development, the surfactant industry has increasingly turned its attention for the search of environmentally safer surfactants from renewable sources to replace petrochemical based products. Fatty acids are anionic surfactants under their deprotonated forms coming from renewable sources and have many advantages due their availability in large amount in nature and their biocompatibility [3]. They could be used for applications in various field from washing, material recovery processes, environmental cleanup to encapsulation and drug delivery. Fatty acid molecules have an aliphatic tail and a polar headgroup. As a function of the medium conditions, the alkyl tail can be in crystalline or liquid state and the headgroup can be in its protonated $(-\mathrm{COOH})$ or deprotonated $\left(-\mathrm{COO}^{-}\right)$ state. Due to these intrinsic properties, fatty acid molecules are responsive surfactants under 
the action of $\mathrm{pH}, \mathrm{CO}_{2}$ and temperature. The modifications occurring at the molecular level under stimuli lead to modifications of the fatty acid self-assembled structures in aqueous solution at the mesoscopic scale. Recently, some studies have focused on the use of the structural transformations of self-assemblies based on fatty acids occurring at the mesoscopic level to finely control the physical properties at the macroscopic scale. Original macroscopic properties have been found, such as thermoresponsive foams and interfaces [4-6], which cannot be achieved by conventional low molecular weight synthetic surfactants.

In the present short topical review, we will describe the recent results which highlight the interest of using fatty acids as responsive surfactants to tune the properties at the macroscopic scale under stimuli. At the outset of this review, we will provide a brief overview on the molecular nature of fatty acids, how to disperse them in water and the general factors at microscopic scale leading to various self-assembled morphologies at mesoscopic scale in dilute aqueous medium. We will then describe how modifications at the molecular level triggered by $\mathrm{pH}, \mathrm{CO}_{2}$ and temperature can tune the self-assemblies based on fatty acids at mesoscopic scale. In the third part, we will focus on the link between these structural transformations and the macroscopic properties: rheological properties, interfacial properties, foam and emulsion stability. In particular, the role of the chain melting process at the molecular level triggered by temperature will be examined in details and demonstrated as a tool to control the responsiveness at the macroscopic scale in a reversible manner.

\section{Fatty acid molecules as anionic surfactants: Fundamentals}

\section{1) Fatty acid at molecular state}

From a chemical structure viewpoint, fatty acids are amphiphilic molecules with an aliphatic tail, which is either saturated or unsaturated, and with a polar headgroup which can be in its protonated $(-\mathrm{COOH})$ or deprotonated $\left(-\mathrm{COO}^{-}\right)$state. The aliphatic tail can have some additional groups such as hydroxyl group [7]. Fatty acids are usually obtained by hydrolysis of oils from various oleochemical sources such as animal, marine and vegetable [3]. The source of the oils determines the composition of the fatty acid mixture. Most of the animal sources are characterized by a high concentration of saturated fatty acids, whereas marine sources such as fish oils provide long chain and unsaturated acids. For oils coming from vegetable sources, the fatty acid composition is linked to the plant origin and sort. Usually, fatty acids are classified as a function of the aliphatic chain length [3]. Fatty acids with 
aliphatic tails fewer than six carbons are called "short-chain" fatty acids. Between 6 and 12 carbons, they are called "medium-chain" fatty acids. From 14 up to 22 carbons, fatty acids are classified as "long-chain" fatty acids. Above 22 carbons in the aliphatic tails, they belong to the "very long chain" fatty acids category. Fatty acids become anionic surfactants under their deprotonated form [3]. It is well recognized that anionic surfactants tend to be biodegradable and less toxic compared to the cationic ones [8]. Fatty carboxylates can be degraded by $\beta$ oxidation and completely mineralized or incorporated into biomass [9]. Fatty acids can be qualified as anionic surfactants with both low toxicity and biodegradability. The main drawbacks to use fatty acid molecules as surfactant are linked to their low solubility in aqueous solution and their high sensitivity to divalent and trivalent ions in solution. For example, alkali ions form strong ion pairs with fatty acids which decrease their solubility in water $[10,11]$.

\section{2) Self-assemblies based on fatty acids in aqueous solution}

Fatty acid properties (solubility, surface activity, self-assembly, etc.) are directly linked to the molecular structure: types and length of the hydrophobic tails, nature of the hydrophilic groups (ionized or non-ionized), presence of additional groups on the alkyl chain and the binding with counter-ions. For all surfactants, the solubility depends on the Krafft point which corresponds to the temperature at which the concentration of maximal solubility equals the critical micellar concentration (CMC) [12]. For fatty acids, the Krafft point is directly linked to the molecular structure and increases rapidly with the increase of the alkyl chain length. This increase of the Krafft point, corresponding to a decrease in solubility, prevents the use of long chain fatty acids in many applications. Various physical-chemistry approaches have been designed to decrease the Krafft point temperature in order to disperse efficiently long chain fatty acids over a wide range of temperatures in aqueous solution [13]. Obviously, chemical modifications of fatty acids can yield to an increase of fatty acid solubility but others methods can be applied without any modification of the fatty acid molecule [3]. More than 40 years ago, Gebicki and Hicks reported the dispersion of oleic acid in vesicles in an aqueous solution [14]. The bilayer of these vesicles is formed by both the non-ionized and ionized forms of the fatty acid molecule. These two forms interact by lateral hydrogen bonding [15]. The ionized form corresponds to the negatively charged surfactant. The formation and stability of the vesicles depends on the protonation/ionization ratio of the terminal carboxylic acid which governs the formation of hydrogen bonds [16]. This approach was then extended to disperse 
various saturated fatty acids under the form of vesicles [16]. Another method consists in mixing fatty acids with cationic surfactants to obtain catanionic systems [17]. The electrostatic interactions between the fatty acid and the oppositely charged cationic surfactant enable to disperse the fatty acid in aqueous solution by giving rise to catanionic surfactant pairs with amphiphilic properties. The use of a large organic counter-ion provides another effective method to enhance the solubility of fatty acids by ion-pairing [18]. An interesting point is that the method used to successfully disperse the fatty acid controls the fatty acid properties such as its limit of solubility and the self-assembly [13]. The self-assembly type can be predict by using the concept based on molecular shape and called the packing parameter defined as $p=\frac{v}{a * l c}$, where $a$ is the effective headgroup area and $V$ is the volume of the hydrophobic chain possessing maximum effective length $l$ [12]. In surfactant systems, it is known that for $p<1 / 3$, there is a tendency to form spherical micelles. For $1 / 3<p<1 / 2$, wormlikes micelles (WLMs) can be present. When $1 / 2<p<1$, bilayers or vesicles are obtained. The nature of the fatty acid and the approach used to disperse it lead to various values of $p$ giving a large variety of fatty acid self-assemblies in aqueous dilute medium ( $>90 \mathrm{wt} . \%$ water) from usual self-assembled morphologies (spherical micelles, worm-like micelles, vesicle and bilayer) to unusual morphologies such as tubes [19], nanodiscs [20], icosahedra [21], buckled membranes [22] or cones [23].

An important parameter to take into account with fatty acid molecules is the $\mathrm{pKa}[24,25]$. The pKa value represents the ionic environment of the solution where $50 \%$ of hydrogen atoms are removed from the carboxylic group by the existing $\mathrm{OH}^{-}$ions in solution [26]. Most of the short-chain fatty acids such as acetic acid (C2) or propionic (C3) acid have a pKa value around 4.8 [26]. The pKa value can increase by increasing the carbon chain length of fatty acids [27]. For example, pKa values around 9 have been found for the palmitic acid (C16) [27]. The increase of the chain length of fatty acids leads to an increase of the hydrophobic interactions between the chains of adjacent molecules, which gives rise to an increase of the $\mathrm{pKa}$ value [26-28]. In the same way, the $\mathrm{pKa}$ value decreases in presence of unsaturation for long chain fatty acids since the intermolecular distance increases and the area per molecule increases [26]. The environmental conditions have a drastic effect on the pKa value. When fatty acid molecules are embedded in the self-assembly, the pKa values are modified. For example, it has been determined for lauric acid (C12) that the pKa value changes from 7.6 to 4.9 when lauric acid is embedded in anionic (SDS) and cationic (DoTAC) micelles, 
respectively [29]. As a result, all the modifications of the environmental conditions such as $\mathrm{pH}$ or temperature have an impact on the $\mathrm{pKa}$ of the fatty acid molecules embedded in the self-assembly, which is important to take into account to produce responsive systems [30,31]. The environmental conditions can tune the fatty acid molecules at the microscopic scale such as the effective headgroup area and effective length of the alkyl chain, which in turn modify the packing parameter. As discussed below, fatty acid self-assembled structures can transit from one morphology to another by modifying the medium conditions such as $\mathrm{pH}, \mathrm{CO}_{2}$ and temperature.

\section{Effect of stimuli at molecular level on the fatty acid self-assembled structures at the mesoscopic scale}

\section{1) $\mathrm{pH}$ effect}

Surfactants responsive to $\mathrm{pH}$ changes are a subject of growing interest owing to their wide potential in novel applications, as $\mathrm{pH}$ variations can control the molecular self-assembly [2]. Fatty acid headgroup has carboxylic group and the $\mathrm{pH}$ of the aqueous medium tunes the degree of ionization between the protonate $(-\mathrm{COOH})$ and deprotonated $\left(-\mathrm{COO}^{-}\right)$forms. Fatty acids are a simple class of $\mathrm{pH}$-responsive surfactants. When the $\mathrm{pH}$ is varied, the effective area of headgroup is modified at the microscopic scale. The deprotonated form possesses a bigger effective headgroup area than the protonated form. The corresponding $p$ value for the deprotonated form is reduced in comparison to the protonated form approaching values close to $1 / 2-1 / 3$, which favors the formation of worm-like or spherical micelles. By finely tuning the $\mathrm{pH}$, the ratio between the protonated and deprotonated forms can be adjusted which modifies in turn the packing parameter, the hydrogen bonding and electrostatic interactions [32]. Just by changing the protonation/ionization ratio of the carboxylic acid, a wide range of fatty acid aggregates can be reversibly obtained at the mesoscopic scale.

It has long been known that saturated or unsaturated fatty acids can form vesicles or liposomes near the apparent $\mathrm{pKa}$ value, where the protonated and deprotonated forms exist in similar fractions leading to the formation of hydrogen bonding between fatty acid molecules. By adding $\mathrm{NaOH}$, the $\mathrm{pH}$ increases leading higher ratio of ionized to protonated molecules and micelles are the dominant self-assembly [14, 33, 34]. When the $\mathrm{pH}$ is decreased by 
adding $\mathrm{HCl}$, oil droplets are obtained due to higher ratio of protonated to ionized molecules. The $\mathrm{pH}$ range for vesicles formation varies as a function of the fatty acid molecules $\mathrm{pKa}$, which is linked to their chemical nature [16]. A modification of the $\mathrm{pH}$ medium by adding $\mathrm{NaOH}$ or $\mathrm{HCl}$ can lead to other kinds of fatty acid self-assemblies than only vesicles or spherical micelles. For example, erucic acid (unsaturated long chain fatty acid), which is one of the most important kind of vegetable $\omega-9$ fatty acid, can be used as pH-responsive surfactant to obtain a $\mathrm{pH}$-switchable worm-like micellar system [35]. The addition of $\mathrm{NaOH}$ leads to the saponification of erucic acid molecules in aqueous solution forming sodium erucate, which is an anionic surfactant (Fig. 1.a).

(a)

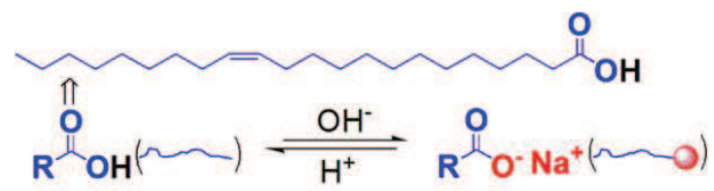

(b)
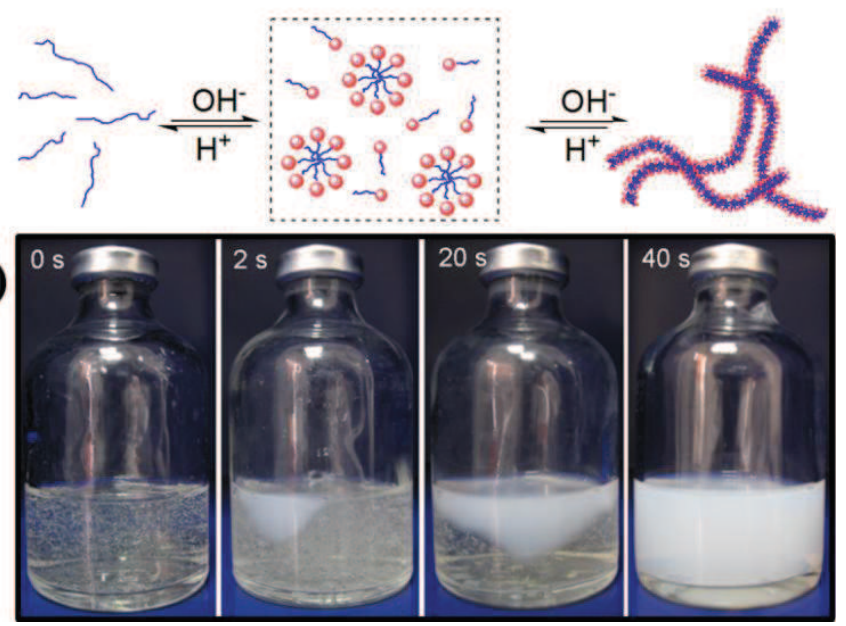

Figure 1: (a) Schematic illutrating the chemical principles of the pH-switchable wormlike micelles obtained with the erucic acid. (b) Snapshots showing the evolution of the $100 \mathrm{mM}$ erucic acid solution as a function of the carbon dioxide bubbling time. Reprinted and adapted from $[35,36]$ with permission.

Under a low $\mathrm{pH}$ environment, only few erucic acid molecules can be neutralized into sodium erucate which can be dissolved in aqueous medium as erucate ions. By increasing progressively the $\mathrm{pH}$, more and more sodium erucate molecules are obtained leading to an increase of surfactant monomers in water. When the concentration of erucate surfactant molecules is high enough, the molecules self-assemble under spherical micelles. By the progressive $\mathrm{pH}$ increase, the aggregates evolve from spherical micelles to WLMs. When the $\mathrm{pH}$ value is decreased, erucate ions are converted again into non-ionic erucic acid molecules leading to the loss of the function of surfactant. The decrease of monomer surfactants disrupts 
progressively the surfactant aggregates. This $\mathrm{pH}$-responsive behavior is reversible when the $\mathrm{pH}$ values are cyclically increased and decreased.

Another class of fatty acid systems called "true" catanionic systems has been extensively studied due to its $\mathrm{pH}$ response. These systems are obtained from a mixture of fatty acids with cationic surfactants in their hydroxide form. The counter-ions used are only $\mathrm{H}^{+}$and $\mathrm{OH}^{-}$and no excess salt is formed by mixing the two surfactants since $\mathrm{OH}^{-}$react with $\mathrm{H}^{+}$to form water [17]. The main parameter in these systems is the molar ratio between the two components. An excess of fatty acid in the mixture means that a part of the fatty acid molecules are still under protonated forms. In an excess of the cationic surfactant in the hydroxide form, all fatty acids are under their deprotonated forms and $\mathrm{OH}^{-}$ions can be present in slight or in large excess in the aqueous solution. A simple change of the molar ratio modifies the $\mathrm{pH}$ of the aqueous medium. In these catanionic systems, a $\mathrm{pH}$ modification leads to a change of both the degree of ionization of the fatty acid and the molecular electrostatic interactions with the cationic component [13]. Many studies have been done by the Hao's research group about fatty acid catanionic systems. For example, in the system composed by lauric acid with trimethyltetradecylammonium hydroxide (TTAOH), in presence of TTAOH in excess, all fatty acid molecules are under deprotonated forms and micelles are obtained at $25^{\circ} \mathrm{C}$ [37]. At equimolarity between the two components, vesicles are present. The transition between micelles and vesicles occurs due to a change of the surface charge density governed by the molar ratio. In the case of the catanionic system obtained with myristic acid and cetyltrimetylammonium hydroxide $(\mathrm{CTAOH})$ various usual and unusal structures have been obtained by modifying the molar ratio [13]. When the cationic surfactant is in large excess, the $\mathrm{pH}$ is high due to an excess of $\mathrm{OH}^{-}$leading to the formation of WLMs. When the catanionic surfactant is in slight excess, corresponding to intermediate $\mathrm{pH}$ values, nanodiscs are formed and the excess of cationic surfactants forms their edges [20]. When the fatty acid is in excess, the $\mathrm{pH}$ decreases and facetted vesicles and icosahedra are obtained due to the segregation of the excess of fatty acid molecules [21].

Recently, studies from Olvera de la Cruz's research group have highlighted how both the selfassembled structures at the mesoscopic scale and the crystalline order within fatty acid bilayers at the microscopic scale can be easily controlled by $\mathrm{pH}$ (by using $\mathrm{NaOH}$ ) in a catanionic fatty acid system $[22,38,39]$. They studied palmitic acid in the presence of a cationic component comprising a trivalent cationic headgroup made of three lysine amino acids associated to a hydrophobic palmitoyl tail [39]. The key parameter in this catanionic 
system is the headgroups stoichiometric ratio. At equimolarity between fatty acid and cationic component, the charge ratio is $+3 /-1$. At low $\mathrm{pH}$, faceted vesicles are obtained with a twodimensional hexagonal molecular arrangement of the ionic molecules inside the bilayer. At this $\mathrm{pH}$, the cations are fully charged and the degree of ionization of the fatty acid is very low. The electrostatic attractions between the headgroups are expected to be weak and the molecular tails pack into a hexagonal lattice. By increasing the $\mathrm{pH}$ to intermediate values, ribbons are obtained with a rectangular $\mathrm{C}$-structure for the molecular arrangement inside the bilayer. At this intermediate $\mathrm{pH}$ range, both the palmitic acid headgroups and the trilysine cationic molecules are expected to be nearly fully charged. This results to an enhancement of electrostatic interactions, leading to a higher packing density of the molecular tails inside the bilayer, which requires orientational ordering of the alkyl tails. The change of electrostatic interactions between the headgroups modifies the packing density which leads to a distortion of the hexagonal lattice into a $2 \mathrm{D}$ rectangular-C lattice. This crystalline transition induced by the electrostatic interactions at the microscopic scale opens the closed membranes of the faceted vesicles into ribbons at the mesoscopic level. At high $\mathrm{pH}$, faceted vesicles are present with a two-dimensional hexagonal lattice as for low $\mathrm{pH}$. At high $\mathrm{pH}$, the degree of ionization of the fatty acid is high and the cation is not fully charged contrary to -low $\mathrm{pH}$ range. The solution $\mathrm{pH}$ tunes the degree of ionization of the amphiphiles, modifying their molecular electrostatic interactions which in turn control the molecular packing and the crystalline phases of the ionic membranes at molecular scale. This results to a change of the selfassembled morphology. The $\mathrm{pH}$-driven ionization of the two amphiphilic components within the bilayers enables to obtain various self-assembled morphologies made of bilayer at the mesoscopic scale: closed unilamellar and multilamellar faceted vesicles, open sheets or ribbons.

\section{2) $\mathrm{CO}_{2}$ as a tool to tune the $\mathrm{pH}$}

A fine variation of $\mathrm{pH}$ is offered by using $\mathrm{CO}_{2}$ with the advantage of avoiding contamination and modification of the medium solution by the addition of other chemical components such as $\mathrm{NaOH}$ or $\mathrm{HCl}$ [36]. Many examples of such control have been published for various types of surfactants [2]. Up to date, only one example is described in the literature on the use of $\mathrm{CO}_{2}$ to tune fatty acid properties as surfactant [36]. The WLMs obtained from erucate solution at high $\mathrm{pH}$ described in the previous section have been used to study the effect of $\mathrm{CO}_{2}$ as a trigger [36]. When $\mathrm{CO}_{2}$ is injected by bubbling into the WLMs solution, carbonic acid is 
produced within a few seconds and the $\mathrm{pH}$ decreases (Fig. 1.b). The $\mathrm{H}+$ concentration increases which favors the formation of erucic acid molecules. By increasing the time of bubbling, more and more anionic surfactant molecules (sodium erucate) are converted into erucic acid molecules decreasing the surfactant molecules concentration. Surfactants are going away from the WLMs which result into a transition from WLMs to spherical micelles or free monomers. Usually, a common way to deplete carbon dioxide is to inject nitrogen by bubbling in order to reversibly switch the systems [40, 41]. However in this system, bubbling nitrogen cannot make a reversible process since the increase of $\mathrm{pH}$ is not high enough to reinitiate the formation of WLMs. This first example using $\mathrm{CO}_{2}$ as a trigger highlights the potential of this simple, inexpensive, non-toxic and biocompatible method to tune selfassemblies based on fatty acids in aqueous medium. This study has established the 'proof-ofconcept' of such control and we believe that $\mathrm{CO}_{2}$ can be used as stimulus for various fatty acid systems. Moreover, the reversibility offered by this trigger by bubbling nitrogen could be achieved in systems for which the structural transition occurs at enough low $\mathrm{pH}$.

\section{3) Temperature effect}

In contrary to other stimuli such as $\mathrm{pH}$, the temperature can lead to reversibility over system characteristics without changes in chemical composition. Temperature is known to strongly affect the fatty acid self-assembled structure due to the chain melting process [13]. "Chain melting" means that the hydrocarbon chains of lipids lose their ordered crystalline state to gain a disordered liquid crystal state. The chain melting occurs above a characteristic temperature called melting transition temperature $T_{m}[42] . T_{m}$ is influenced by many factors such as the alkyl chain length, $\mathrm{pH}$ value, ion binding, etc. [43-45]. This melting process between the crystalline and the liquid state can occur at a precise temperature or span over a wide range of temperatures. $\mathrm{T}_{\mathrm{m}}$ corresponds to the beginning of the melting process. In the case of a progressive chain melting over a wide range of temperatures, the chain melting process begins at $T_{m}$ and the crystallized chains melt progressively until all the chains reach the liquid state. This progressive melting can be determined by coupling Differential Scanning Calorimetry (DSC) and Wide angle X-ray scattering (WAXS) experiments [13]. The chain melting process can induce reversible phase structural transitions. When the melting process occurs at a precise temperature, a single structural self-assembly modification is observed. In the case of a progressive chain melting, consecutive and complex multiple structural modifications can occur at the mesoscopic scale. The importance of the chain melting process 
on fatty acid self-assembled structures can be illustrated by the 12-hydroxystearic acid (12HSA) multilamellar tubes system [19]. The 12-HSA tubes of micron length are obtained via ion-pairing of the 12-hydroxystearic acid monomers with various alkanolamines [46]. For equimolar ratio between 12-HSA and ethanolamine, the chain melting process is progressive [13]. This phenomenon begins at $\mathrm{T}_{\mathrm{m}} \sim 47^{\circ} \mathrm{C}$ and finishes at around $70^{\circ} \mathrm{C}$. Below $\mathrm{T}_{\mathrm{m}}$, the 12HSA tubes average dimensions are $10 \mu \mathrm{m}$ in length and $0.6 \mu \mathrm{m}$ in diameter. Such tubes exhibit multiple morphological structural transitions with temperature due to the progressive chain melting process. The outer tube diameter starts to rapidly increase when the melting process begins $\left(\mathrm{T}_{\mathrm{m}} \sim 47^{\circ} \mathrm{C}\right)$, reaching its maximum value of $\sim 3-5 \mu \mathrm{m}$ around $50^{\circ} \mathrm{C}$ (Fig. 2 ). Then, the tubes diameter decreases again upon a further increase of temperature back to the same initial value $(D \sim 0.6 \mu \mathrm{m})$ probably in mixture with spherical micelles [19]. Finally, at the end of the melting process $\left(\mathrm{T} \sim 70^{\circ} \mathrm{C}\right)$, when 12-HSA chains are all in liquid state, only spherical micelles are present in solution.

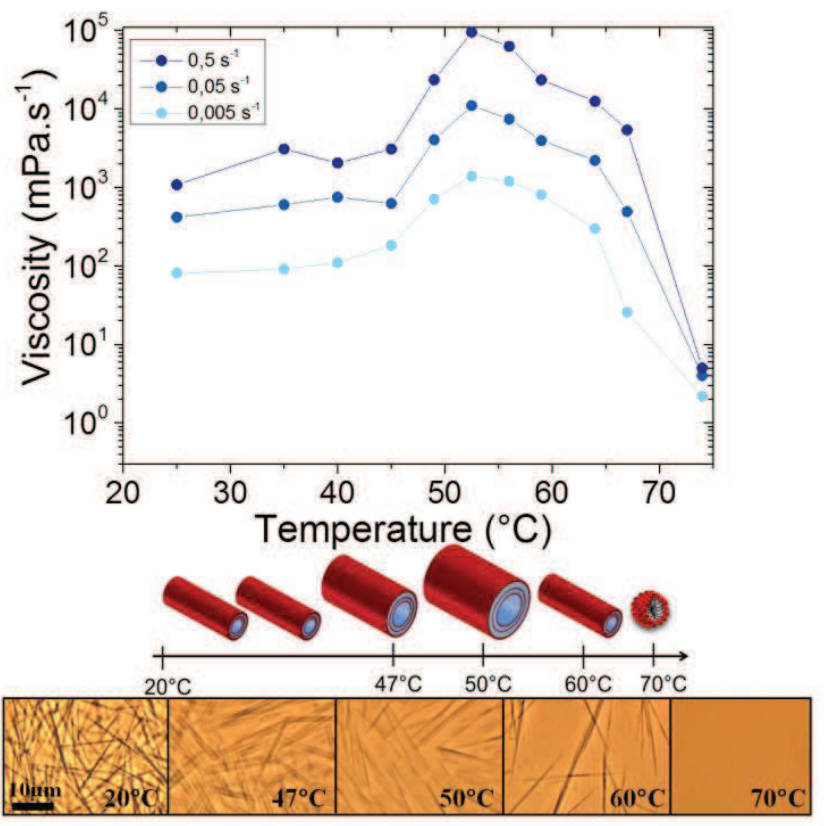

Figure 2: Evolution of the viscosity of the 12-HSA tubes solution as a function of temperature for three different shear rates. The 12-HSA tubes structural evolution occurring in bulk with temperature is illustrated by the schematic and the corresponding pictures obtained by phase contrast microscopy. Adapted from [46, 47] with permission from American Chemical Society and The Royal Society of Chemistry. Copyright 2011 American Chemical Society. 
All these structural modifications between micron size tubes with temperature tunable diameter and spherical micelles triggered by the chain melting are completely reversible. For 12-HSA tubes, $\mathrm{T}_{\mathrm{m}}$ can be easily tuned by the medium conditions (ionic strength, $\mathrm{pH}$ and nature of the alkanolamines) [46, 48]. In excess of ethanolamine, the chain melting process occurring for the 12-HSA tubes occurs at a unique temperature [13]. 12-HSA tubes transit directly into spherical micelles at $\mathrm{T}_{\mathrm{m}}\left(\mathrm{T}_{\mathrm{m}} \sim 42^{\circ} \mathrm{C}\right)$ without any change of the tubes diameter. Similar structural transitions triggered by temperature have been obtained in salt free fatty acid catanionic systems such as transitions from vesicles to lamellar networks [44] or facetted to unilamellar vesicles [43]. Depending on the molar ratio between fatty acid and cationic surfactant, the melting process can be progressive, spanning over a large range of temperatures, or occurring at a single well-defined temperature [43].

Another effect of the temperature inducing structural transitions at the mesoscopic scale is linked to counter-ion binding. This effect has been illustrated in the catanionic system obtained from lauric acid and TTAOH. For concentration of lauric acid higher than 3.25 w.t $\%$, when TTAOH is in excess, lamellar phases are obtained at room temperature. This excess of cationic component leads to the presence of small quantity of free $\mathrm{OH}^{-}$counterions in solution [49]. Some of the $\mathrm{OH}^{-}$ions can exist near the bilayer and create a negative charge lowering the surface charge of the bilayer. By increasing the temperature, these negative charges can progressively move away from the bilayer which can in turn be more positively charged. This continuous motion of the $\mathrm{OH}^{-}$counter-ions increases the curvature by modifying the packing parameter and induces the transition from lamellar phases to micelles. With exactly the same system but at lower lauric acid concentration, the presence of an excess of TTAOH yields to a transition between micelles and vesicles by increasing the temperature. In this case, the mechanism at the origin of this structural transition is not linked to a modification of counter-ion binding but can be explained by the variation of components involved in the self-assemblies. At room temperature, micelles are present containing an excess of cationic surfactant. TTAOH has a much higher CMC than the surfactant ion pair formed by TTA+ and lauric acid molecule under deprotonated form. This higher CMC for the cationic component can lead to a gradually dissociation of TTAOH in solution by increasing temperature. This dissociation process decreases the surface charge density of the aggregates modifying the packing parameter which in turn induces a transition from spherical micelles to vesicles [49]. 
Temperature can lead to different modification inside the self-assembly at the molecular scale: chain melting, counter-ion binding and variation of the components concentration. All these phenomena triggered by temperature then induce reversible structural transitions at the mesoscopic scale.

\section{Responsive self-assemblies based on fatty acids: effect on the macroscopic properties}

4.1 Solution rheological properties : effect of chain melting with temperature

All the structural transformations occurring at the mesoscopic scale described in Section 3 under the action of $\mathrm{pH}, \mathrm{CO}_{2}$ and temperature might modify the rheological properties. Indeed, it is well known that solutions of spherical micelles yield low viscosities, whereas the formation of WLMs or vesicles can have strong effects on the rheological properties providing in some cases highly viscous solutions $[35,36,50,51]$. In literature, most of the reported studies deal with single structural modification induced by an external stimulus leading to only one major change in the rheological properties. However, multiple structural transformations can occur with temperature for fatty acid aggregated structures due to the progressive chain melting process spanning over a wide range of temperatures. This progressive phenomenon occurring at molecular level leads to non-trivial and even nonmonotonous changes in the rheological properties of the solution. The changes occurring in the rheological properties during the progressive chain melting process have been studied for two systems: the facetted vesicles obtained from the catanionic mixture made of myristic acid and $\mathrm{CTAOH}$ [43] and the 12-HSA self-assembled tubular structures obtained with ethanolamine as counter-ion in equimolar ratio [47]. In the case of the facetted vesicles, the progressive chain melting begins at $\mathrm{T}_{\mathrm{m}} \sim 43^{\circ} \mathrm{C}$ and finishes at $53^{\circ} \mathrm{C}$ as determined by DSC [43]. This process induces a transition from facetted vesicles towards unilamellar vesicles (Fig. 3.a). Below and above this temperature range $\left(43^{\circ} \mathrm{C}-53^{\circ} \mathrm{C}\right)$, the bulk viscosity is low (Fig. 3.b). In the melting transition region, there is a strong increase in the viscosity within a few degrees. This increase in bulk viscosity can be explained in terms of structural reorganization and metastable coexistence of liquid and crystalline domains in faceted vesicles bilayers at the microscopic scale (Fig. 3.c). 


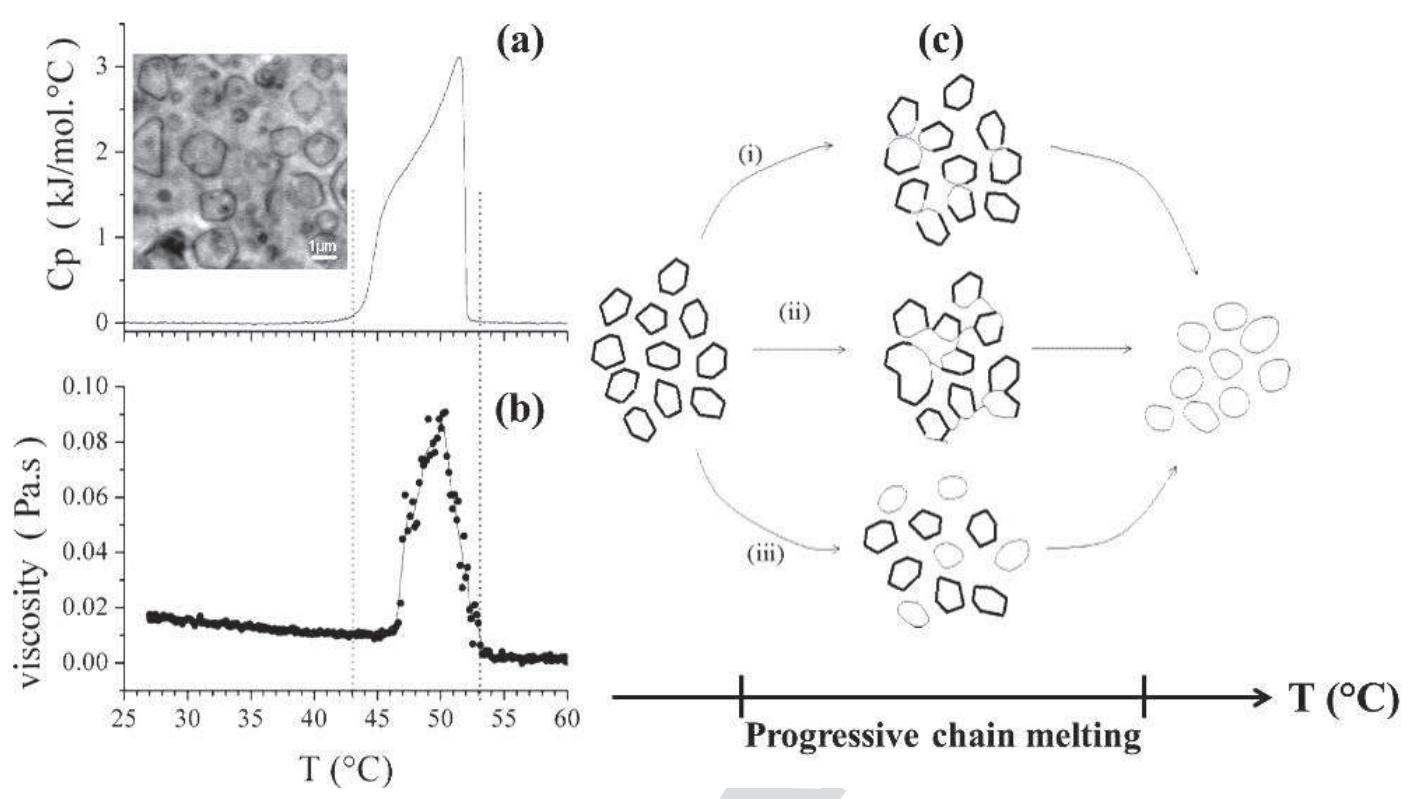

Figure 3: (a) Heat capacity scan obtained by DSC showing the progressive chain melting occuring from $43^{\circ} \mathrm{C}$ to $53^{\circ} \mathrm{C}$ leading to the transition between faceted vesicles to unilamellar vesicles. (b) Evolution of the bulk viscosity as a function of temperature measured at $6.28 \mathrm{rad} \mathrm{s}^{-1}$ of catanionic vesicles $(\Phi=0.1 \mathrm{wt} \%$ ). (c) Schematic showing the possible intermediate states between facetted polyhedra and unilamellar vesicles occurring during the chain melting and at the origin of the rheological behavior. The thick lines symbolize the ordered chains, while the fine, curved lines stand for the disordered chains. Adapted from [43] with permission. Copyright 2004 American Chemical Society.

For the 12-HSA tubes with a temperature tunable diameter which transit into spherical micelles at around $70^{\circ} \mathrm{C}$, the multiple transformations due to the progressive chain melting from $47^{\circ} \mathrm{C}$ to $70^{\circ} \mathrm{C}$ lead to unusual non-monotonous evolution of the bulk viscosity in this range of temperature (Fig. 2) [47]. The fine tuning of the tubes diameter with temperature and the transition from micron-size tubes to spherical micelles at high temperature leads to several rheological behaviors ranging from a simple shear-thinning fluid, to a yield stress fluid or to a Newtonian fluid; note that such strong changes are obtained within a relatively small temperatures range (from 47 to $70^{\circ} \mathrm{C}$ ) [47]. It is important to notice the closed link between the chain melting process occurring at the molecular scale level inside the self-assembled structures with these drastic modifications of the rheological behavior at the macroscopic scale. These two examples highlight how reversible non-monotonous changes of rheological properties can be easily triggered by temperature from simple self-assembled structures based on fatty acid molecules. 


\subsection{Interfacial properties: effect of $\mathrm{pH}$ and temperature}

It is well known that $\mathrm{pH}$ has a drastic effect on the characteristics of fatty acids monolayer self-organized at the air/water interface [52]. As in bulk, a $\mathrm{pH}$ modification of an aqueous phase covered by a fatty acid monolayer leads to changes of the fatty acid molecules ionization state [27]. At low $\mathrm{pH}$, the adsorbed layer contains fatty acid molecules under protonated forms whereas at high $\mathrm{pH}$, fatty acid molecules at the interface are totally deprotonated. When the molecules are deprotonated, ionic repulsions between the polar groups occur and increase the intermolecular distance and the area per molecule. At pH close to the $\mathrm{pKa}$, there is a coexistence of the two forms (deprotonated and protonated) at the interface. They interact by hydrogen bonding and decrease the intermolecular distance. Consequently, the interfacial properties evolve with $\mathrm{pH}$ due to a change of interactions between the molecules. The surface tension, which is linked to the molecular packing and the molecular area at the interface, is consequently tuned by the $\mathrm{pH}[53]$.

Similar effects have been observed at the oil/water interface. The link between surface tension and $\mathrm{pH}$ have been used to produce self-propelled oil droplets from fatty acids molecules (Fig. 4.a) [54]. To obtain these self-propelling droplets, two phases are in contact: oil droplets containing oleic anhydride precursor and an aqueous phase containing fatty acid micelles at alkaline $\mathrm{pH}$. At the droplet interface, when a patch of fresh oil becomes exposed to the water, the hydrolysis of the oleic anhydride precursor occurs. In the presence of alkaline aqueous solution, the oleic anhydride precursor is hydrolyzed to form two oleic acid molecules. The oleic acid molecules produced through hydrolysis within a basic environment result in acidification of the environment close to the oil droplet. A local $\mathrm{pH}$ gradient is self-generated in the solution close to the interface affecting the interfacial tension between oil and water. Such difference in the interfacial tension leads to the motion of the oil droplet. The droplet moves away from this acid product into fresh unmodified alkaline water zone. This system perfectly illustrates the $\mathrm{pH}$ effect on fatty acid molecules at interfaces which can lead to motion at the macroscopic scale. 


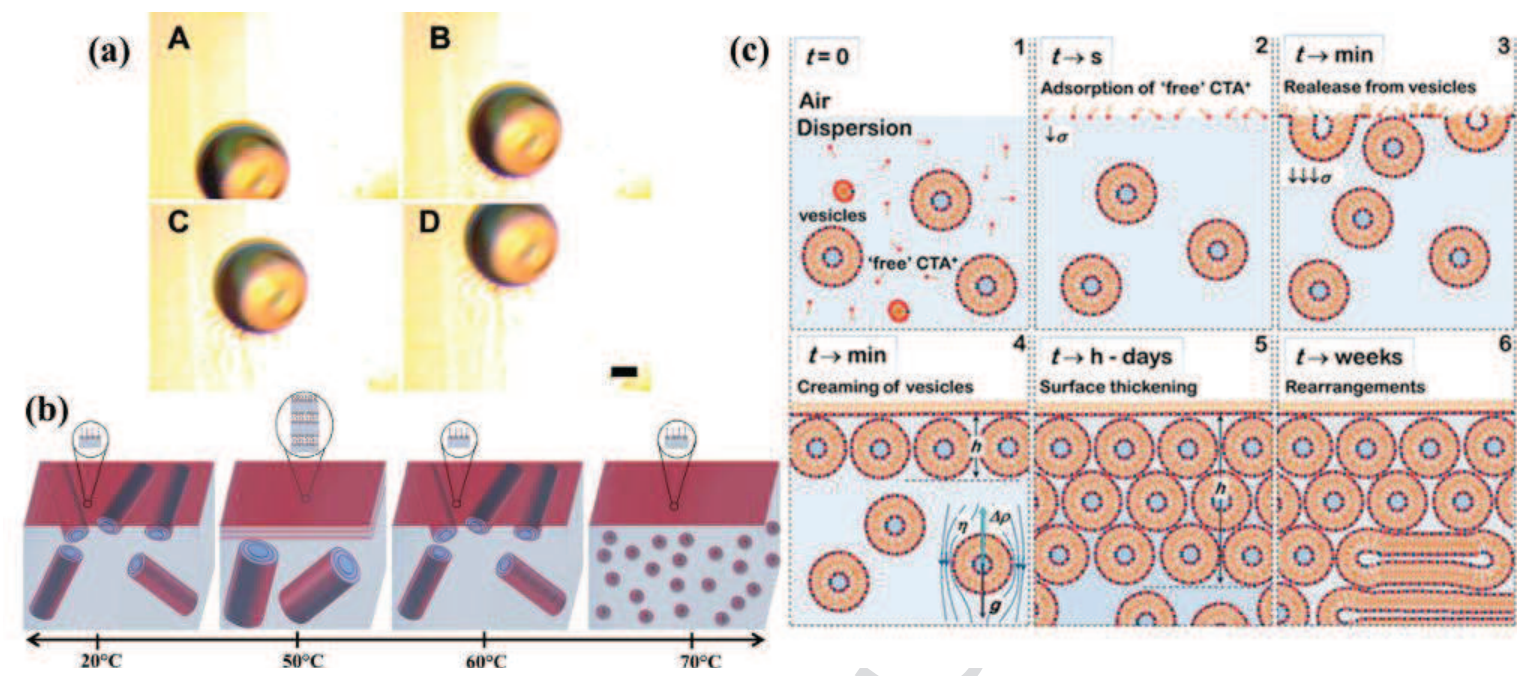

Figure 4: (a) Self-propelling droplet motion observed by phase contrast microscopy (A-D). The droplet moves from the bottom to the top and each frame is captured at $2 \mathrm{~s}$ intervals. The scale bar is $100 \mu \mathrm{m}$. Reproduced from [54] with permission. Copyright 2007 American Chemical Society. (b) Schematic illustration of the structural and dynamic properties of the 12-HSA tubes at the air/water interface with temperature determined by neutron reflectivity experiments. Reproduced from [4] with permission. Copyright 2011 American Chemical Society. (c) Schematic illustrating the adsorption phenomenon occurring in catanionic vesicles obtained with myristic acid with time. Reproduced from [6] with permission. Copyright 2013 American Chemical Society.

This $\mathrm{pH}$ response concerns only the interfacial properties of fatty acid monolayers, but it has been highlighted recently that some self-assembled structures are also able to adsorb at the air/water interface below a monolayer, forming thick layer of few hundred nanometers $[4,6]$. The properties of these aggregated structures at the interface can be tuned by temperature due to the chain melting process, as described in the previous section for the bulk properties. The 12-HSA tubular system with a temperature tunable diameter (see part 3.3) illustrates this phenomenon. By neutron reflectivity experiments, it has been shown that the 12-HSA tubes can adsorb at the air/water interface below a fatty acid monolayer with similar structural characteristics than in bulk [4]. The presence of the very dense monolayer with low equilibrium surface tension $(25 \mathrm{mN} / \mathrm{m})$ protects the tubes and they are not destroyed when they come to the interface. The monolayer is very dense because of strong intermolecular interactions induced by hydrogen bonding between the hydroxy groups of the 12-HSA molecule [47]. An increase of temperature induces the same structural modifications of 12HSA tubes at the interface than in bulk which arise from the progressive chain melting process (Fig. 4.b). Upon heating, when the chain melting process begins, the tubes diameter increases in bulk, leading to an increase of the tubes diameter at the interface. When tubes 
begin to be in closed contact to each other at the interface, they unfold to form a multilamellar layer. Upon further heating, the tubes diameter decreases in bulk and tubes are reformed at the interface. At high temperature, when the chain melting process finishes, the tube-to-micelle transition occurs in bulk and only the 12-HSA monolayer remains at the interface. Tubes can form again just by decreasing the temperature. The structural modifications of the 12-HSA tubes adsorbed at the interface with temperature are reversible and have an impact on the interfacial properties [47]. The viscoelastic properties are directly linked to the aggregated structures adsorbed at the interface, which are controlled by the progressive chain melting process occurring at molecular level. The presence of the tubes below the fatty acid monolayer confers a "solid-like" behavior, but when tubes are unfolded or tubes transit into micelles a "fluid-like" behavior is observed. This system has several reversible consecutive modifications between "solid-like" to "fluid-like" regimes in a few degrees. Another system made of myristic acid and cetyltrimethylammonium chloride, which forms catanionic vesicles in bulk, has been shown to exhibit thermoresponsive interfacial properties [6]. The formation of a very dense and very elastic catanionic monolayer with a low equilibrium surface tension $(29 \mathrm{mN} / \mathrm{m})$ at the interface prevents the vesicles rupture yielding to the presence of several layers of intact vesicles underneath (Fig. 4.c). At low temperature, vesicles bilayers are in crystalline state and extremely rigid with both high compression and bending moduli conferring a solid-like behavior at the interface. Around the chain melting transition temperature of the vesicle bilayers in bulk, the transition between the "solid-like" to "fluid-like" behavior occurs at the interface. Above $\mathrm{T}_{\mathrm{m}}$, the catanionic monolayer and the vesicles layers lost their solid and rigid characteristics inducing changes of the interfacial dynamical properties. This temperature-dependent behavior at the interface is again completely reversible. From these two examples, it seems that the presence of a dense monolayer is a prerequisite to allow and preserve self-assembled structures localized in the vicinity of an interface. The thermoresponsive behavior at the interface highlights the importance of the chain melting phenomenon at molecular scale which gives reversible structural transitions both in bulk and at the interface.

\section{$\underline{4.3 \mathrm{pH}-\text { and Thermo-responsive Foams }}$}

An aqueous foam is a dispersion of gas bubbles in a fluid phase, usually water. A foam is a thermodynamically unstable system and any foam vanishes with time. The foam is destabilised by three coupled mechanisms: drainage, coalescence, and coarsening. Stable 
foams correspond to foams for which these destabilization mechanisms are drastically slowed down. Responsive foams are foams for which the stability can be switched from low to high stability under an external stimulus. One of the prerequisite to obtain a foam is to have a stabilizer which can adsorb at the gas/liquid interface. Fatty acids are surfactants known to easily produce foams. As detailed previously, the interfacial properties of fatty acids can be triggered by $\mathrm{pH}$ which has been shown to affect the foamability and foam stability [27]. The optimal foaming properties are obtained for $\mathrm{pH}$ close to the $\mathrm{pKa}$ since the fatty acid monolayer is dense and close packed at the interface, due to hydrogen bonding. A pH modification leads to changes of fatty acid ionization states both in bulk and at the interface which tunes the foaming properties at the macroscopic scale. For example, from oleate/oleic acid solution, micelles are present at high $\mathrm{pH}$ leading to weak foam stability [55]. At low $\mathrm{pH}$, most of the fatty acid molecules are under protonated forms giving oil droplets in bulk and a foam is almost impossible to produce. The fatty acid oil droplets lead to the almost instantaneous destruction of the film between bubbles by acting as defoamers. At intermediate $\mathrm{pH}$ close to $\mathrm{pKa}$, both the presence of vesicles in bulk inside the foam liquid channels and the dense layer at the interface produce relatively stable foams. Thus, a simple change of the $\mathrm{pH}$ solution modify the foamability and foam stability [55]. However, this method does not induce reversible changes of the foam stability and the solution composition is modified by addition of either base or acid component.

Recently, responsive foams reacting to external stimuli and made from solutions containing self-assemblies based on fatty acids have been reported. These foams are based on the structural transitions occurring both in bulk and at the interface with temperature in a few seconds $[5,56]$. Thermoresponsive foams have been obtained from the 12-HSA tubes described previously. Foams produced from these 12-HSA tubes solution are outstandingly stable over months and described as "ultra-stable" [5]. The origin of this stability comes from both the presence of tubes in the foam liquid channels and at the air/water interface, which prevent the foam destabilization by reducing drainage, coalescence and coarsening [5]. These tubes transit into micelles when the chain melting process, triggered by temperature, is over. Fatty acid micelles are known to give foams with weak stability [57]. By using the temperature-response of this self-assembly both in bulk and at the interface, the production of responsive foams has been for the first time achieved. This phenomenon is shown in the Figure 5.a. 


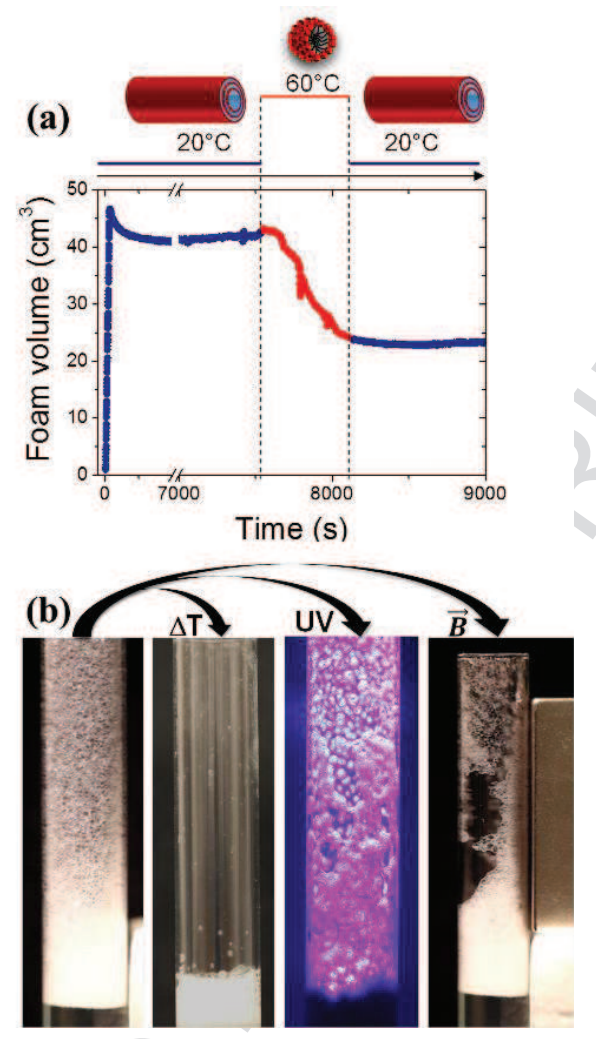

Figure 5: (a) Evolution of the foam volume as a function of the temperature for foam produced with 12-HSA tubes showing the thermoresponse of the foam. Foams are ultrastable when tubes are present inside the foam liquid channels. The tubes to micelle transition at $60^{\circ} \mathrm{C}$ induced a drastic decrease of the foam volume. By decreasing the temperature at $20^{\circ} \mathrm{C}$, tubes are reformed in the foam and the foam volume remains constant again with time. Reproduced from [5] with permission. (b) Illustration of the ability of 12-HSA foam with magnetic particles to respond to different external stimuli: temperature, UV irradiation and magnetic field. Reproduced from [56] with permission from The Royal Society of Chemistry.

Foams produced at $20^{\circ} \mathrm{C}$ are stable due to the presence of the 12-HSA tubes. Upon heating the foam above the tubes-to-micelles transition temperature, the foam volume decreases quickly. The foam is less stable in the absence of tubes. Remarkably, by decreasing the foam temperature below the transition temperature between tubes and micelles, tubes are reformed and the foam destabilization is completely stopped. The ultra-stable behavior is recovered. This phenomenon is almost instantaneous and completely reversible. The origin of the foam responsiveness at macroscopic scale is directly linked to the modification of self-assembled structures at the mesoscopic scale triggered by temperature, which is in turn controlled by the alkyl chain state at the molecular level. Later, to obtain responsive foams from this system but avoiding the direct contact with the foam, light has been used as external stimulus. Indeed, it is known that light offers significant advantages as stimulus compared to the temperature. 
Light can be applied at a precise location without direct contact with the foam. In this aim, the 12-HSA tubes have been combined with carbon black particles (CPB) which are able to convert light stimulus to heat by photothermal conversion effect [56]. Both CPB and 12-HSA tubes are trapped in the liquid channels leading to ultrastable foams. Under UV or solar illumination, CPB particles absorb light causing an increase in temperature inside the foam liquid channels above the temperature transition from tubes to micelles. This results into very fast foam destabilization (Fig. 5.b). The foam can be destabilized multiple times without modification of the system that guaranteed the reversibility. This approach leading to photothermo-responsive foams has been extended to magnetic particles giving rise to the first foams exhibiting stability which can be tuned by three stimuli: temperature, light and magnetic field (Fig. 5.b).

Up to date, it is the first example of foams produced from self-assemblies based on fatty acids for which the stability can be switched reversibly from stable to unstable under external stimuli. However, other fatty acid aggregated structures could be efficient to produce similar foams [58-61]. For example, foams produced from myristic acid and cetyltrimethylammonium chloride have already been studied at room temperature [58]. In this case, foams are very stable due to the presence of catanionic vesicles both in the foam liquid channels and in the films separating bubbles. These vesicles can adsorb at the interface and exhibit thermoresponsive behavior due to the chain melting process [6]. All these information let us assume that responsive foams could be obtained from these catanionic vesicles and from other systems which could exhibit similar features.

\subsection{From thermoresponsive foams to thermoresponsive emulsions?}

Contrary to foaming properties, only one fatty acid aggregated system has been shown to adsorb at oil/water interface leading to the long term stabilization of emulsion at room temperature $[62,63]$. The Zemb's research group has produced the first Pickering emulsion stabilized by catanionic discs of myristic acid and CTAOH. These discs act as particles at the interface which results to the emulsion stabilization. As described previously for various systems at the air/water interface, the discs first rupture in the presence of a bare interface leading to the formation of dense monolayer. Then, discs adsorb and stack below this monolayer. These discs are known to transit into other aggregated structures with temperature due to the chain melting process in aqueous solution [20]. From these observations, it can be 
expected changes of emulsion stability triggered by the chain melting process with temperature. However, this hypothesis has still to be demonstrated.

\section{Summary, conclusion and outlook}

This review brings evidence that fatty acid molecules can be used as natural and responsive surfactants to tune aggregation structure, viscosity, surface activity and foam stability. Most of the recent studies deal with $\mathrm{pH}$ or temperature as stimulus. A $\mathrm{pH}$ modification can be obtained by using $\mathrm{CO}_{2}$ as trigger which seems to be very promising. In all cases, the change occurring at molecular level, either the ionization state of the headgroup or the state of the alkyl chains, leads to drastic modifications of the properties at the mesoscopic and macroscopic scales. It is important to point out that the responsiveness at macroscopic scale, such as demonstrated for foams, is obtained due to the link between the microscopic and the mesoscopic scales.

The use of sodium and potassium carboxylates surfactants is widespread, especially in household and personal cleaning products. The response obtained under stimuli from fatty acid co-assembly systems described in this review could be used for specific applications in various fields from washing, material recovery processes and environmental clean-up. These systems can be highly biocompatible which strongly supports their applicability in customer end products [64]. Some of the systems described in this review are already studied to be used for encapsulation and drug delivery $[65,66]$. It is clear that a major part of self-assemblies based on fatty acids research for years to come will be focus on the use of fatty acids assemblies as soft responsive particles with specific properties.

\section{Acknowledgments}

The authors acknowledge the COST (MP 1106) for support. INRA and Région Pays de la Loire are gratefully acknowledged for Ph.D. grant of A. Arnould. 


\section{$\underline{\text { References }}$}

[1] Theato P, Sumerlin BS, O'Reilly RK, Epps III TH. Stimuli responsive materials. Chemical Society Reviews. 2013;42:7055-6.

[2] Brown P, Butts CP, Eastoe J. Stimuli-responsive surfactants. Soft Matter. 2013;9:2365-74.

[3] Johansson I, Svensson M. Surfactants based on fatty acids and other natural hydrophobes. Current Opinion in Colloid \& Interface Science. 2001;6:178-88.

[4] Fameau A-L, Douliez J-P, Boue F, Ott F, Cousin F. Adsorption of multilamellar tubes with a temperature tunable diameter at the air/water interface. Journal of Colloid and Interface Science. 2011;362:397-405.

[5] Fameau A-L, Saint-Jalmes A, Cousin F, Houssou BH, Novales B, Navailles L, et al. Smart Foams: Switching Reversibly between Ultrastable and Unstable Foams. Angewandte ChemieInternational Edition. 2011;50:8264-9.

[6] Arriaga LR, Varade D, Carriere D, Drenckhan W, Langevin D. Adsorption, organization and rheology of catanionic layers at the air/water interface. Langmuir. 2013;29:3214-22.

[7] Fameau A-L, Gaillard C, Marion D, Bakan B. Interfacial properties of functionalized assemblies of hydroxy-fatty acid salts isolated from fruit tomato peels. Green Chemistry. 2013;15:341-6.

[8] Vlachy N, Merle C, Touraud D, Schmidt J, Talmon Y, Heilmann J, et al. Spontaneous formation of bilayers and vesicles in mixtures of single-chain alkyl carboxylates: effect of $\mathrm{pH}$ and aging and cytotoxicity studies. Langmuir. 2008;24:9983-8.

[9] Steber J, Berger H. Biodegradability of anionic surfactants. Biodegradability of surfactants: Springer; 1995. p. 134-82.

[10] McBain JW, Vold RD, Frick M. A Phase Rule Study of the System Sodium Stearate/Water. The Journal of Physical Chemistry. 1940;44:1013-24.

[11] Klein R, Kellermeier M, Drechsler M, Touraud D, Kunz W. Solubilisation of stearic acid by the organic base choline hydroxide. Colloids and Surfaces a-Physicochemical and Engineering Aspects. 2009;338:129-34.

[12] Israelachvili J. Intermolecular and surface forces. Second Edition ed. London Academic press limited; 1992.

[13] Fameau A-L, Zemb T. Self-assembly of fatty acids in the presence of amines and cationic components. Advances in Colloid and Interface Science. 2014.

[14] Gebicki JM, Hicks M. Ufasomes are stable particles surrounded by unsaturated fatty acid membranes. Nature. 1973;243:232-4.

[15] Apel CL, Deamer DW, Mautner MN. Self-assembled vesicles of monocarboxylic acids and alcohols: conditions for stability and for the encapsulation of biopolymers. Biochimica et Biophysica Acta (BBA) - Biomembranes. 2002;1559:1-9.

[16] Morigaki K, Walde P. Fatty acid vesicles. Current Opinion in Colloid \& Interface Science. 2007; 12:75-80.

[17] Hao J, Hoffmann H. Self-assembled structures in excess and salt-free catanionic surfactant solutions. Current Opinion in Colloid \& Interface Science. 2004;9:279-93.

[18] Klein R, Touraud D, Kunz W. Choline carboxylate surfactants: biocompatible and highly soluble in water. Green Chemistry. 2008;10:433-5.

[19] Douliez JP, Pontoire B, Gaillard C. Lipid tubes with a temperature-tunable diameter. Chemphyschem. 2006;7:2071-3.

[20] Zemb T, Dubois M, Deme B, Gulik-Krzywicki T. Self-assembly of flat nanodiscs in saltfree catanionic surfactant solutions. Science. 1999;283:816-9. 
[21] Dubois M, Demé B, Gulik-Krzywicki T, Dedieu J-C, Vautrin C, Désert S, et al. Selfassembly of regular hollow icosahedra in salt-free catanionic solutions. Nature. 2001;411:672-5.

[22] Greenfield MA, Palmer LC, Vernizzi G, Cruz MOdl, Stupp SI. Buckled membranes in mixed-valence ionic amphiphile vesicles. Journal of the American Chemical Society. 2009;131:12030-1.

[23] Douliez JP. Self-assembly of hollow cones in a bola-amphiphile/hexadiamine salt solution. Journal of the American Chemical Society. 2005;127:15694-5.

[24] Shinoda K. The effect of alcohols on the critical micelle concentrations of fatty acid soaps and the critical micelle concentration of soap mixtures. The Journal of Physical Chemistry. 1954;58:1136-41.

[25] Liptak MD, Shields GC. Accurate $\mathrm{p} \mathrm{K}$ a calculations for carboxylic acids using complete basis set and Gaussian-n models combined with CPCM continuum solvation methods. Journal of the American Chemical Society. 2001;123:7314-9.

[26] Kanicky JR, Shah DO. Effect of Degree, Type, and Position of Unsaturation on the $p<i>$ $\mathrm{K}</ \mathrm{i}><\mathrm{sub}>\mathrm{a}</ \mathrm{sub}>$ of Long-Chain Fatty Acids. Journal of Colloid and Interface Science. 2002;256:201-7.

[27] Kanicky JR, Poniatowski AF, Mehta NR, Shah DO. Cooperativity among molecules at interfaces in relation to various technological processes: Effect of chain length on the pK(a) of fatty acid salt solutions. Langmuir. 2000;16:172-7.

[28] Tanford C. The Hydrophobic Effect: Formation of Micelles and Biological Membranes 2d Ed: J. Wiley.; 1980.

[29] da Silva FLB, Bogren D, Söderman O, Åkesson T, Jönsson B. Titration of fatty acids solubilized in cationic, nonionic, and anionic micelles. Theory and experiment. The Journal of Physical Chemistry B. 2002;106:3515-22.

[30] Koynova R, Caffrey M. An index of lipid phase diagrams. Chemistry and Physics of Lipids. 2002;115:107-219.

[31] Cevc G, Seddon JM, Hartung R, Eggert W. Phosphatidylcholine-fatty acid membranes. I. Effects of protonation, salt concentration, temperature and chain-length on the colloidal and phase properties of mixed vesicles, bilayers and nonlamellar structures. Biochimica et Biophysica Acta (BBA)-Biomembranes. 1988;940:219-40.

[32] Morrow BH, Koenig PH, Shen JK. Self-Assembly and Bilayer-Micelle Transition of Fatty Acids Studied by Replica-Exchange Constant $\mathrm{pH}$ Molecular Dynamics. Langmuir. 2013;29:14823-30.

[33] Cistola DP, Hamilton JA, Jackson D, Small DM. Ionization and Phase-Behavior of Fatty-Acids in Water - Application of the Gibbs Phase Rule. Biochemistry. 1988;27:1881-8.

[34] Hargreaves WR, Deamer DW. Liposomes from ionic, single-chain amphiphiles. Biochemistry. 1978;17:3759-68.

[35] Zhang Y, Han Y, Chu Z, He S, Zhang J, Feng Y. Thermally induced structural transitions from fluids to hydrogels with $\mathrm{pH}$-switchable anionic wormlike micelles. Journal of Colloid and Interface Science. 2013;394:319-28.

[36] Zhang Y, Yin H, Feng Y. CO 2-responsive anionic wormlike micelles based on natural erucic acid. Green Materials. 2014;2:95-103.

[37] Li H, Hao J. Phase behavior and rheological properties of a salt-free catanionic surfactant TTAOH/LA/H2O system. The Journal of Physical Chemistry B. 2008;112:10497-508.

[38] Leung C-Y, Palmer LC, Kewalramani S, Qiao B, Stupp SI, de la Cruz MO, et al. Crystalline polymorphism induced by charge regulation in ionic membranes. Proceedings of the National Academy of Sciences. 2013;110:16309-14. 
[39] Leung C-Y, Palmer LC, Qiao BF, Kewalramani S, Sknepnek R, Newcomb CJ, et al. Molecular Crystallization Controlled by $\mathrm{pH}$ Regulates Mesoscopic Membrane Morphology. ACS nano. 2012;6:10901-9.

[40] Zhang Y, Feng Y, Wang Y, Li X. CO2-Switchable Viscoelastic Fluids Based on a Pseudogemini Surfactant. Langmuir. 2013;29:4187-92.

[41] Zhang Y, Chu Z, Dreiss CA, Wang Y, Fei C, Feng Y. Smart wormlike micelles switched by CO 2 and air. Soft Matter. 2013;9:6217-21.

[42] Schneider MF, Marsh D, Jahn W, Kloesgen B, Heimburg T. Network formation of lipid membranes: triggering structural transitions by chain melting. Proceedings of the National Academy of Sciences. 1999;96:14312-7.

[43] Vautrin C, Zemb T, Schneider M, Tanaka M. Balance of $\mathrm{pH}$ and ionic strength influences on chain melting transition in catanionic vesicles. Journal of Physical Chemistry B. 2004;108:7986-91.

[44] Shen Y, Hao J, Hoffmann H, Wu Z. Reversible phase transition from vesicles to lamellar network structures triggered by chain melting. Soft Matter. 2008;4:805-10.

[45] Michina Y, Carriere D, Charpentier T, Brito R, Marques EF, Douliez JP, et al. Absence of Lateral Phase Segregation in Fatty Acid-Based Catanionic Mixtures. Journal of Physical Chemistry B. 2010;114:1932-8.

[46] Fameau A-L, Cousin F, Navailles L, Nallet Fdr, Boué Fo, Douliez J-P. Multiscale structural Characterizations of fatty acid multilayered tubes with a temperature-tunable diameter. The Journal of Physical Chemistry B. 2011;115:9033-9.

[47] Fameau A-L, Saint-Jalmes A. Yielding and flow of solutions of thermoresponsive surfactant tubes: tuning macroscopic rheology by supramolecular assemblies. Soft Matter. 2014.

[48] Fameau AL, Houinsou-Houssou B, Novales B, Navailles L, Nallet F, Douliez JP. 12Hydroxystearic acid lipid tubes under various experimental conditions. Journal of Colloid and Interface Science. 2010;341:38-47.

[49] Li H, Wieczorek SA, Xin X, Kalwarczyk T, Ziebacz N, Szymborski T, et al. Phase transition in salt-free catanionic surfactant mixtures induced by temperature. Langmuir. 2009;26:34-40.

[50] Han Y, Chu Z, Sun H, Li Z, Feng Y. "Green" anionic wormlike micelles induced by choline. RSC Advances. 2012;2:3396-402.

[51] Sun W, Shen Y, Hao J. Phase Behavior and Rheological Properties of Salt-Free Catanionic TTAOH/DA/H2O System in the Presence of Hydrophilic and Hydrophobic Salts. Langmuir. 2010;27:1675-82.

[52] Schulman J, Hughes A. On the surface potentials of unimolecular films. Part IV. The effect of the underlying solution and transition phenomena in the film. Proceedings of the Royal Society of London Series A. 1932;138:430-50.

[53] Wen XY, Lauterbach J, Franses EI. Surface densities of adsorbed layers of aqueous sodium myristate inferred from surface tension and infrared reflection absorption spectroscopy. Langmuir. 2000;16:6987-94.

[54] Hanczyc MM, Toyota T, Ikegami T, Packard N, Sugawara T. Fatty acid chemistry at the oil-water interface: Self-propelled oil droplets. Journal of the American Chemical Society. 2007;129:9386-91.

[55] Shu X, Meng Y, Wan L, Li G, Yang M, Jin W. pH-Responsive Aqueous Foams of Oleic Acid/Oleate Solution. Journal of Dispersion Science and Technology. 2014;35:293-300.

[56] Fameau A-L, Lam S, Velev OD. Multi-stimuli responsive foams combining particles and self-assembling fatty acids. Chemical Science. 2013;4:3874-81. 
[57] Fameau A-L, Ventureira J, Novales B, Douliez J-P. Foaming and emulsifying properties of fatty acids neutralized by tetrabutylammonium hydroxyde. Colloids and Surfaces A: Physicochemical and Engineering Aspects. 2012;403:87-95.

[58] Varade D, Carriere D, Arriaga L, Fameau A-L, Rio E, Langevin D, et al. On the origin of the stability of foams made from catanionic surfactant mixtures. Soft Matter. 2011;7:6557-70. [59] Fameau A-L, Houinsou-Houssou B, Ventureira J, Navailles L, Nallet F, Novales B, et al. Self-Assembly, Foaming, and Emulsifying Properties of Sodium Alkyl Carboxylate/Guanidine Hydrochloride Aqueous Mixtures. Langmuir. 2011;27:4505-13.

[60] Novales B, Navailles L, Axelos M, Nallet F, Douliez JP. Self-assembly of fatty acids and hydroxyl derivative salts. Langmuir. 2008;24:62-8.

[61] Novales B, Riaublanc A, Navailles L, Houssou BH, Gaillard C, Nallet F, et al. SelfAssembly and Foaming Properties of Fatty Acid-Lysine Aqueous Dispersions. Langmuir. 2010;26:5329-34.

[62] Schelero N, Lichtenfeld H, Zastrow H, Möhwald H, Dubois M, Zemb T. Single particle light scattering method for studying aging properties of Pickering emulsions stabilized by catanionic crystals. Colloids and Surfaces A: Physicochemical and Engineering Aspects. 2009;337:146-53.

[63] Schelero N, Stocco A, Möhwald H, Zemb T. Pickering emulsions stabilized by stacked catanionic micro-crystals controlled by charge regulation. Soft Matter. 2011;7:10694-700.

[64] Klein R, Müller E, Kraus B, Brunner G, Estrine B, Touraud D, et al. Biodegradability and cytotoxicity of choline soaps on human cell lines: effects of chain length and the cation. RSC Advances. 2013;3:23347-54.

[65] Béalle G, Lartigue L, Wilhelm C, Ravaux J, Gazeau F, Podor R, et al. Surface decoration of catanionic vesicles with superparamagnetic iron oxide nanoparticles: a model system for triggered release under moderate temperature conditions. Physical Chemistry Chemical Physics. 2014.

[66] Salerno C, Chiappetta DA, Arechavala A, Gorzalczany S, Scioscia SL, Bregni C. Lipidbased microtubes for topical delivery of Amphotericin B. Colloids and Surfaces B: Biointerfaces. 2013;107:160-6. 


\section{Highlights:}

- Review of fatty acid as responsive surfactant

- Correlation between molecular state and self-assemblies structural transitions triggered by $\mathrm{pH}, \mathrm{CO}_{2}$ and temperature

- Link between self-assemblies based on fatty acids and corresponding macroscopic properties under stimuli

- Chain melting process as prerequisite to obtain thermoresponsive interfaces and foams 